Published in final edited form as:

Exp Eye Res. 2007 September ; 85(3): 381-392. doi:10.1016/j.exer.2007.06.002.

\title{
Preservation of outer retina and its synaptic connectivity following subretinal injections of human RPE cells in the Royal College of Surgeons rat
}

\author{
Isabel Pinilla ${ }^{1,2}$, Nicolás Cuenca ${ }^{1,3}$, Yves Sauvé ${ }^{1,4}$, Shaomei Wang ${ }^{1,5}$, and Raymond D \\ Lund ${ }^{1,5}$, $^{*}$ \\ ${ }^{1}$ Moran Eye Center, Department of Ophthalmology and Visual Sciences, University of Utah, Salt \\ Lake City, UT. USA. \\ 2 Servicio de Oftalmología, Hospital Universitario Miguel Servet, Isabel la Católica 1-3, Zaragoza, \\ 50009 Spain. Instituto Aragonés de Ciencias de la Salud.
}

${ }^{3}$ Departamento de Fisiología Genética y Microbiología, Universidad de Alicante, Alicante, 03080 Spain.

${ }^{4}$ Departments of Ophthalmology and Physiology, 7-55 Medical Sciences Bldg, University of Alberta, Edmonton AB T6G 2H7, Canada

${ }^{5}$ Department of Ophthalmology, Casey Eye Institute, Oregon Health and Science University, Portland OR. USA.

\section{Abstract}

We have examined how transplantation of an RPE cell line to the subretinal space of RCS rats affects the distribution of synaptic connectivity markers in the outer plexiform layer of the retina. Using markers of pre- and post-synaptic profiles (bassoon and synaptophysin as presynaptic markers and mGluR6 for postsynaptic profiles) we found that the normal orderly patterns seen between photoreceptors and rod and ON-cone bipolar cells were severely disrupted in dystrophic rats. In areas in which injected cells preserved photoreceptors, more normally appearing pairing of pre- and postsynaptic markers was seen for both rods and cones. The degree of normality correlated with the amount of photoreceptor rescue. The secondary changes that are normally seen in bipolar and horizontal cells were prevented by the photoreceptor preservation. ERG recordings in the animals subsequently studied morphologically showed that both a- and b-waves could be rescued by grafting, albeit with lower amplitudes than normal. Together these anatomical and physiological studies indicate that besides the integrity of outer nuclear layer cells and phototransduction processes, relay circuitry through the outer retina was rescued by cell grafts.

\footnotetext{
(C) 2007 Elsevier Ltd. All rights reserved.

* Corresponding author: Raymond D. Lund, Department of Ophthalmology, Casey Eye Institute, Oregon Health and Science University, Portland OR 93739-3098.

Publisher's Disclaimer: This is a PDF file of an unedited manuscript that has been accepted for publication. As a service to our customers we are providing this early version of the manuscript. The manuscript will undergo copyediting, typesetting, and review of the resulting proof before it is published in its final citable form. Please note that during the production process errors may be discovered which could affect the content, and all legal disclaimers that apply to the journal pertain.
} 


\section{INTRODUCTION}

The RCS rat has a primary defect in retinal pigment epithelial (RPE) cells and serves as a model for exploring potential treatment approaches for the retinal dystrophy that occurs in humans caused by RPE dysfunction. One approach involves subretinal injection of healthy RPE cells or cell lines prior to significant degenerative changes to preserve photoreceptors ( $\mathrm{Li}$ and Turner, 1988, Gouras et al., 1989, Sheedlo et al., 1991, Lund et al., 2001, Wang et al., 2005). However, the anatomical presence of photoreceptors does not guarantee that they can transduce light signals or that normal relay pathways within the retina can be sustained. Indeed, electroretinogram (ERG) studies suggest that despite the photoreceptor rescue, there are deficiencies in ERG responsiveness (Pinilla et al., 2005, Sauvé et al., 2004, Sauvé et al., 2006), and there is evidence in other transplantation and growth factor delivery studies of disconnection between outer nuclear layer integrity and ERG and other functional responses (Spencer et al., 2001, Bok et al., 2002, Girman et al., 2005).

To examine how grafts may affect patterns of circuitry associated with photoreceptor rescue we have built on our previous immunocytochemical studies (Cuenca et al., 2005), which detailed how connectivity patterns in the outer plexiform layer deteriorate in the RCS rat. Here we have focused on the effects of subretinal transplantation of the RPE cell line, ARPE-19, on the connectivity patterns shown by these synaptic markers in the plexiform layers and correlate directly with the changes seen in full field illumination electroretinogram responses. This adds to a body of work studying the effects of such cell transplantation on overall organization of the retina (Wang et al., 2005) and on centrally mediated visual functions (Lund et al., 2001, Coffey et al., 2002, Sauvé et al., 2002, 2004, McGill et al., 2004, Girman et al., 2003) by examining two specific issues cone morphology and the degree to which connections formed by the rescued photoreceptors are sustained. Cone morphology was important to study in detail because previous work (Girman et al., 2005) emphasized that cone survival rather than overall outer nuclear layer thickness may be the important index of success. Furthermore the apparent absence of rod function at low luminance found in those studies examining light and dark adaptation even after transplantation raises a concern as to whether there is a defect at the level of phototransduction or in circuitry within the retina.

We report the presence of relatively normal connectivity patterns between photoreceptors and ON bipolar cells suggesting that therapies designed to limit the progress of photoreceptor loss also serve to sustain substrates of synaptic connectivity in the retina and therefore should permit relay of information through the retina.

\section{METHODS}

\section{Animals}

A total of 14 dystrophics RCS rats $\left(\mathrm{rdy}^{+} \mathrm{p}^{+}\right)$were used for this study. Three congenic RCS rats $\left(\mathrm{rdy}^{-} \mathrm{p}^{+}\right)$at postnatal day (P) 30 were used as controls. All animals were bred in a colony at the University of Utah, and maintained under a 12-hour light/dark cycle (light cycle mean illumination: $30 \mathrm{~cd} / \mathrm{m}^{2}$ ). The animals were housed and handled with the authorization and supervision of the Institutional Animal Care and Use Committee at the University of Utah. Experiments were carried out in accordance with the guidelines laid down by the NIH regarding the care and use of animals for experimental procedures (NIH Publications No. 80-23, revised 1996). The procedures also conformed to the ARVO Statement for the Use of Animals in Ophthalmic and Vision Research. All efforts were made to minimize the number of animals used and their discomfort. 


\section{Cell preparation and injection}

At age P22, 8 dystrophic rats received trans-scleral subretinal injections of a suspension of cells of the human RPE cell line, ARPE-19 (available from the ATCC, American Tissue Culture Collection; reference number: CRL-2302) as previously described (Lund et al., 2001). The donor cells have been characterized in previous works (Kanuga et al., 2002, Klimanskaya et al., 2004). Injections were made to the mid-temporal region of the right retina. Six further rats served as unoperated controls for comparison purposes. Half of the untreated and transplanted rats were sacrificed at P90 and the other half at P120. Four dystrophic RCS rats received a trans-scleral injection of medium alone. Based on previous works where sham ERG preservation was limited to P60 (Sauvé et al. 2006), the 4 rats were sacrificed at this age: at the later time points, sham injections give ERG responses, similar to untreated rats.

All rats, transplanted, sham and control, received intraperitoneal dexamethasone injections, given daily for 14 days $(85 \mathrm{mg} / \mathrm{kg})$; in addition cyclosporin A was added to the drinking water ( $210 \mathrm{mg} / \mathrm{l}$; resulting blood concentration: 250-300 $\mu \mathrm{g} / \mathrm{l}$ ) (Coffey et al., 2002) for the length of the experiment to limit rejection responses to the donor cells.

\section{ERG recordings}

Animals were prepared under dim red light following overnight dark adaptation, and ERGs were recorded as previously described (Pinilla et al., 2004, Sauvé et al., 2006). At P90, ERGs were recorded for the $8 \mathrm{hRPE}$-injected and 6 unoperated dystrophic RCS rats, after which, half of the animals in each group was used for anatomy. At P120, ERGs were again recorded from the remaining half. Non-dystrophic congenic rats provided normal records for comparison

\section{Immunostaining}

Animals were anesthetized with a lethal dose of urethane (12.5 g/kg i.p.) and perfused transcardially with phosphate buffered saline (PBS). Eyes were enucleated. The eyecups were fixed in 4\% paraformaldehyde in 0.1 M PBS at $\mathrm{pH} 7.4$ for $1 \mathrm{~h}$ and then washed in $0.1 \mathrm{M}$ PBS before being cryoprotected in 10\% sucrose for $1 \mathrm{~h}, 20 \%$ sucrose for $1 \mathrm{~h}$ and $30 \%$ sucrose overnight at $4{ }^{\circ} \mathrm{C}$. The following day, they were embedded in OCT and $10 \mu \mathrm{m}$ thick sections were cut on a cryostat in a horizontal plane (to ensure that the area of retina containing the grafted cells was cut perpendicularly), and mounted on glass slides. Sections were treated as in a previous study (Cuenca et al., 2004) for immunostaining, using a range of primary antibodies, either singly or in combination as detailed in Table 1. Slides were mounted in watermount (Vector Labs) and coverslipped for viewing by confocal microscopy (Zeiss LSM 510). Pinholes were $77 \mu \mathrm{m}$ and the widths of optical sections were $0.9 \mu \mathrm{m}$. Final images were obtained from the projections of 4 to 7 single frames. TIFF images were enhanced using Adobe Photoshop software. To control for non-specific staining, some sections were stained omitting the primary antibody.

The photoreceptor row number was counted in at least four different pictures of all the studied animals. The number of rows is expressed as the mean \pm SD.

\section{RESULTS}

\section{Morphological studies}

Baseline observations-A series of previous studies (Cuenca et al., 2005, Wang et al., 2005, summarized in Fig. 1) provide the background for the present observations and are briefly detailed.

Low magnification images of the retinas in unoperated P90 dystrophic RCS rat retinas showed the ONL P90 dystrophic RCS rat with a single layer of photoreceptors in the mid-temporal 
retina (Fig 1a, red channel). This is the region of retina which is rescued by cell injections in the experimental rats: in the treated animals, an extended area of photoreceptor preservation was seen extending from the injection site both at P90 (Fig 1b, red channel) and P120 (Fig 1c, red channel). In areas distant from the injection site, the morphology of the photoreceptors resembled untreated dystrophic RCS rat retinas (Fig 1b, 1c, red channel).

By comparison, normal congenic RCS rats showed 12-14 layers of photoreceptors (mean 13.25 \pm 0.93 ) (Fig2a, red channel). Cones, stained with gamma-transducin, had cell bodies localized in the outer-most part of the layer and well developed inner and outer segments (Fig $2 \mathrm{~b}$, green channel).

ARPE-19 grafts preserved a photoreceptor layer up to 7-8 cells deep at P90 (mean value 7.00 \pm 0.63 ) (Fig 1b, 2c, red channel. The syringe in the inset shows the injection site. The square shows where the picture was taken) and 5-6 rows at P120 (mean value 5.66 \pm 0.81 ) (Fig 1c, $2 \mathrm{e}$, red channel): these had well developed outer segments. Cones appeared similar to normal, although their outer segments were shorter (Fig 2d, 2f, green channel). Away from the area of best rescue at P90 and 120, the number of preserved photoreceptors decreased and their morphology became more like that of age-matched unoperated dystrophic rats (Fig $2 \mathrm{~g}, 2 \mathrm{~h}$, red channel).

Rod bipolar cells were stained using antibodies against protein kinase $\mathrm{C} \alpha(\mathrm{PKC}-\alpha)$. These cells in treated animals were quite similar to those seen in normal non-dystrophic rats (Fig 3a, green channel), and clearly different from age-matched dystrophics. At this age, in dystrophic retinas, (Fig 1a, Fig 3b, green channel), rod bipolars, in retinal areas corresponding to the area of the graft, showed dendritic sprouting into the debris zone as was described previously (Cuenca et al., 2005): in the areas where the debris zone had already disappeared, bipolar cells could be found with severe dendritic atrophy. In treated animals, rod bipolar dendrites were normally configured within the area of optimal photoreceptor rescue and there was no evidence of dendritic sprouting (Fig 3c, 3e, green channel). Moving away from the area of maximum preservation, bipolar cell dendrites were seen sprouting into the debris zone (Fig 3d, green channel) or a disappearance of their dendrites (Fig 3f, green channel).

Photoreceptor morphology and antigenicity-In order to study the expression of the different cone opsins, we stained the transplanted retinas with antibodies against blue and redgreen opsins. Red-green opsins were expressed in the area of photoreceptor preservation mainly in cone outer segments. The segments were shorter and thicker than in non-dystrophic rats (Fig 4b vs 4a). In the transplanted retinas cone outer segment, cell body, axon and pedicle were easily recognized. The opsin was expressed in the entire cell instead of being limited to the segments as in normal congenic rats (Fig 4b, green channel). Blue cones, which normally represent a very small proportion of all cones, were seen in small numbers in the area of preservation (data not shown). Distant from the area of graft protection, most of the remaining photoreceptor outer segments were stained with an antibody against red-green opsins: only very occasional cells were blue opsin-positive. These cells in contrast to cones in the area of maximum graft protection presented a marked abnormal morphology with a short axon and disruption of the pedicle as well as of outer and inner segments (red-green cone Fig 4d, blue cone Fig 4c, green channel).

Rods were also preserved in the area of maximum photoreceptor protection. In 120 day old treated retinas, rhodopsin immunoreactivity was no longer confined to the outer segments of the remaining rods, but also encompassed the cell bodies (Fig 4e, red channel), even after transplant-induced rescue. They were only rarely found at more distant locations. 
Connectivity markers in the outer plexiform layer-We have explored whether connectivity markers associated with rods and cones appeared normal in the outer plexiform layer in the area of photoreceptor preservation.

Presynaptic markers for photoreceptor terminals: bassoon and synaptophysin paired with mGluR6 and PKC a-Synaptophysin is a presynaptic marker that stains the whole presynaptic photoreceptor profile. When used in conjunction with PKC staining there was a continuous band of label, associated with the dendritic tips of the rod bipolar cells in the area of photoreceptor preservation (Fig 3c, arrows, red channel). Away from the preserved area, this pattern was lost and synaptophysin was only distributed as a discontinuous band (Fig. $3 \mathrm{~d}$, red channel). In areas where the debris zone persisted, rod bipolar cells in P90 dystrophic retinas had profuse dendritic sprouting associated with scattered synaptophysin profiles within both the OPL and debris zone (Fig 3d, red channel).

Bassoon is a presynaptic protein, which in the OPL, labels the synaptic ribbons of cone and rod terminals. In the area associated with a transplant, pairing between bassoon and the dendritic tips of the rod bipolar cells (PKC-immunoreactivity) was clearly seen (Fig 3e, red channel). Remote from the region of optimal photoreceptor rescue, bassoon immunoreactivity was further diminished, becoming patchy, particularly in the periphery where rod bipolar cells had lost their dendrites (Fig 3f, red channel).

Bassoon staining was also paired with metabotropic glutamate receptor 6 (mGluR6), which labels the postsynaptic receptors on the dendritic tips of both rod and cone ON-bipolar cells.

In non-dystrophic rats, bassoon staining was paired in two ways with mGluR6 staining, either as a band of regularly arrayed paired punctate staining profiles in the outer OPL (Fig 5a, green channel), corresponding to rod spherules partnered with the dendritic tips of the associated rod ON-bipolar cells or with the postsynaptic profiles appearing as disk-like formations, located more proximally in the OPL (Fig 5a arrowheads, green channel), corresponding to ON-cone bipolar cells receiving input from cone pedicles.

In retinas from dystrophic RCS rats at P90, bassoon antibody staining showed spots scattered throughout the OPL, which were rarely seen paired with mGluR6-stained profiles (Fig 5b, green channel). MGluR6 immunoreactivity was diminished and distributed as patches in the region of remaining dendrites even where they had sprouted into the debris zone (Fig $5 \mathrm{~b}$, green channel): it also stained cell bodies and even the axons of ON-bipolar cells. Sham injected animals at P60 showed a few more spared pairs of these synaptic markers, but they failed to form a continuous layer. There were no remarkable differences between the region immediately adjacent to the injection site (Fig 5c) and more distant (Fig 5d). The results were compared to those seen in unoperated P60 dystrophic RCS rats (see Cuenca et al., 2005).

In transplanted eyes in, pairing between bassoon and mGluR6 (Figs 5e, $5 \mathrm{~g}$ ) was sustained in the area of maximum photoreceptor protection, although the number of pairs was clearly diminished compared with normal retinas in congenic RCS rats (Fig 5a). Both cone and rod pairing with their ON-bipolars could be seen. Neither on-bipolar cell bodies nor axons were stained (Fig 5e, 5g), although such staining was seen distant from the area of graft preservation (Figs 5f, 5h).

Remote from the region of optimal photoreceptor preservation, bassoon immunoreactivity was further diminished, becoming patchy, although on rare occasion, pairing between bassoon and rod bipolar-related mGluR6 stained profiles could still be found (Figs 5f, 5h) 
Presynaptic markers for photoreceptor terminals paired with calbindin staining for horizontal cells-There was a clear relationship between bassoon and horizontal cell dendrite tips in non-dystrophic rats (Fig 6a; bassoon is in the red channel, calbindin in the green channel) that was missing in dystrophics. In the dystrophic animals, horizontal dendrites showed a clear sprouting into the debris zone and bassoon staining in the OPL was diminished (Fig 6b). A few bassoon dots could be found inside the debris zone. In areas preserved by the injected cells (Fig 6c), the relationship between bassoon staining and horizontal cell dendrites was similar to that seen in non-dystrophic rats (Fig 6a). Practically all the dendritic tips had bassoon staining associated with them. Sprouting outside the OPL was infrequent (Fig 6c). Away from the area of optimal photoreceptor preservation, bassoon immunoreactivity was clearly diminished (Fig 6d), and in the periphery distant from the graft, some horizontal cells had very few processes (Fig 6e) while others sprouted processes into the debris zone, although this was less than in age-matched dystrophics (Fig $6 f$ vs fig 6b).

\section{ERG findings}

While an exhaustive study of ERG findings after RPE transplants has been published elsewhere (Sauvé et al., 2006), it was important to explore in the very same retinas exactly how the anatomical preservation of photoreceptor to bipolar cell synapses correlated with the activation of retina circuits postsynaptic to photoreceptors as reflected by the ERG b-wave. Figure 7 shows a representative example of the difference in ERG responsiveness between a treated and an untreated RCS rat at P90.

In previous work (Sauvé et al., 2006) we found that in medium-injected dystrophic rats (sham group), no scotopic b-wave was recordable at $\mathrm{P} 90$ and by $\mathrm{P} 120$; the b-waves were replaced by negative deflections.

In rats having received subretinal cell injections, there was clear preservation of scotopic bwaves and oscillatory potentials both at P90 and P120. Clear evidence for rod contribution to post-synaptic activation in cell-injected retinas comes from the observation that $b$-waves were elicited with flash intensities below cone thresholds $\left(<-1.22 \mathrm{log} \mathrm{cds} / \mathrm{m}^{2}\right.$, see Figure 7).

Additional evidence comes from a recent detailed ERG study using a double flash protocol to extract the rod contribution top the mixed scotopic b-wave (Sauvé et al., 2006; which was beyond the scope of the present study). This analytical approach showed that intensity response series in which b-waves were elicited below cone thresholds also had rod contributions to mixed response, i.e. at flash intensities exceeding cone thresholds. Finally, the results obtained here with photopic responses demonstrate the preservation of cone-driven b-waves in cellinjected rats. Therefore, ERG recordings indicate that subretinal hRPE cell injections preserved both the rod and cone-driven post-synaptic activation of the inner retina circuitry. Such preservation was only seen in hRPE cell injected retinas, which were the very same retinas in which anatomical preservation of synapses between photoreceptor and bipolar cells were documented.

\section{DISCUSSION}

The present study shows that preservation of photoreceptors by introduction of ARPE-19 cells to the subretinal space also serves to sustain the substrates of synaptic connectivity, especially associated with rod circuitry within the outer plexiform layer. It is clear also that besides significant retention of rods, specific cone subtypes are sustained, albeit with some reduction in the thickness of the outer segment length. Correlated with these morphological findings, scotopic ERG responses are sustained, although not as well as might be predicted by the morphological observations. Sustained photopic ERG responses are however in accord with the continued presence of cones at 120 days of age. 
The work builds on a background of studies detailing first the progress of photoreceptor loss in the RCS rat (Bourne et al., 1938, Dowling \& Sidman, 1962, Cuenca et al., 2005), how cell patterns change or are sustained during degeneration and preservation and what effect photoreceptor loss and survival have on centrally mediated visual function, studied both physiologically and behaviorally (Girman et al., 2005, LaVail 2001, Li \& Turner, 1988, Lin et al, 1996, Little et al, 1996, Lund et al., 2001, 2003, McGill et al., 2004, Sauvé et al., 2004, Sheedlo et al., 1991, Vollrath et al., 2001, Wang et al., 2005).

The observations showed that without cell transplantation, ERG response patterns followed the course of photoreceptor loss quite closely, although acuity studies (measured under photopic conditions), showed a slower progression of deterioration

(McGill et al., 2004). Somewhat surprisingly dark and light adaptation studies showed no evidence of function at low luminance levels, suggesting that rods are not functioning normally if at all (Girman et al., 2005). This is in accord with previous work (Perlman 1978, Perlman 1978b) showing phototransduction defects in young dystrophic RCS rats. The adaptation studies did suggest that with respect to luminance thresholds, cone function continued normally at first but then gradually deteriorated over several months. So the possibility is presented of rod photoreceptors that are unable to perform normal phototransduction and that quickly lose connection with bipolar and horizontal process in the OPL. Previous work (Peng et al., 2003) has indicated that the rod bipolar cells might in fact receive ectopic input from remaining cone photoreceptors. While there is indication that with time there are substantial changes in inner retinal organization (Jones et al., 2003, Marc et al., 2003, Pignatelli et al., 2004), which may in part be the result of vascular pathology (Villegas-Pérez et al., 1998) but which has little to say for the early detailed circuit reorganization during the time course studied here.

Previous work examining patterns of rescue has shown the pattern of donor cell distribution, and how this relates to rescue within the ONL. While there is some loss of acuity compared with non-dystrophic rats, it is not nearly as devastating as in untreated rats, but the dark and light adaptation studies (Girman et al., 2005) have shown that retinal function at low luminance levels is still compromised. This would appear to contradict the present observations indicating relatively normal circuitry and raises the possibility that while rods may still not be effective in normal phototransduction, they sustain connections with the inner retina. ERG studies do show some activity attributable to rod function for a period, although this is not sustained indefinitely. Both a- and b-waves were rescued beyond the time when they would normally disappear in untreated or sham-injected animals, albeit with reduced amplitudes but similar scotopic mixed a-wave/b-wave amplitude ratios at P90 as in non-dystrophic rats. Sustaining this ratio could imply that activity from the preserved photoreceptors is conveyed to downstream circuitry with a similar efficacy as in the non-dystrophic retina. The reduced amplitudes most likely reflects the limited area of rescue (previous work using the same technology has reported rescue involving up to 30\% of the retinal area; Sauvé et al., 2004, Wang et al., 2005). The deterioration in this ratio by P120, suggests that the OPL relays are less efficient by this time point. Fewer pre- and post-synaptic pairings in the outer plexiform layer may be important. There may also be instability in rod function, as reflected in the finding that a-wave amplitudes, at P90 in treated animals, are extremely variable between consecutive flashes.

The results show that the photoreceptor morphology and antigen expression patterns are relatively normal, although rod numbers are reduced from normal. The configuration of synaptic markers is also preserved in the outer plexiform layer through the area preserved by the transplants, but in reduced numbers. The aberrant sprouting seen in untreated retinas is contained in the area of rescue. 
These observations collectively suggest that while the substrates of rod function are sustained in the outer plexiform layer by transplantation, this is not sufficient to guarantee normal rod function. Phototransduction may be compromised, but how or whether the sustained OPL circuitry affects retinal function in the absence of normal rod input function clearly deserves attention. Is there functional plasticity of the kind summarized by Vaney (Vaney, 2002). Is there systematic synaptic reorganization resulting from the abnormal input function? These issues deserve further attention.

\section{ACKNOWLEDGMENTS}

The authors would like to thank Dr. James F. McGinnis, (University of Oklahoma) for providing the recoverin antibodies, Dr. Robert Molday, (University of British Columbia) for providing the rhodopsin antibody, Dr. Bin Lu for injecting the cells into the subretinal space of RCS rats and Mrs. Xiaoying Cheng for her technical assistance.

Grant information:

This work was supported by NIH (EY14038), Wynn Foundation, and FFB. Dr. Pinilla and Dr. Cuenca were supported by grants from the Spanish Government (FIS BA03/0016, PI042399 and BFU2006-00957/BFI), ONCE and FUNDALUCE. Dr. Lund is a recipient of a Research to Prevent Blindness Senior Scientific Investigator Award.

\section{REFERENCES}

Bok D, Yasumura D, Matthes MT, et al. Effects of adeno-associated virus-vectored ciliary neurotrophic factor on retinal structure and function in mice with a P216L rds/peripherin mutation. Exp. Eye. Res 2002;74:719-35. [PubMed: 12126945]

Coffey PJ, Girman S, Wang SM, et al. Long-term preservation of cortically dependent visual function in RCS rats by transplantation. Nat. Neurosci 2002;5:53-56. [PubMed: 11753416]

Cuenca N, Pinilla I, Sauvé Y, Wang S, Lu B, Lund RD. Regressive and reactive changes in the connectivity patterns of rod and cone pathways of $\mathrm{P} 23 \mathrm{H}$ transgenic rat retina. Neuroscience 2004;127:301-317. [PubMed: 15262321]

Cuenca N, Pinilla I, Sauvé Y, Lund RD. Changes in synaptic connectivity following progressive photoreceptor degeneration in RCS rats. Eur. J. Neurosci 2005;22:1057-1072. [PubMed: 16176347]

Girman SV, Wang S, Lund RD. Cortical visual functions can be preserved by subretinal RPE cell grafting in RCS rats. Vis. Res 2003;43:1817-1827. [PubMed: 12826105]

Girman SV, Wang S, Lund RD. Time course of deterioration of rod and cone function in RCS rat and the effects of subretinal cell grafting: a light- and dark-adaptation study. Vis. Res 2005;45:343-354. [PubMed: 15607350]

Gouras P, Lopez R, Kjeldbye H, Sullivan B, Brittis M. Transplantation of retinal epithelium prevents photoreceptor degeneration in the RCS rat. Prog. Clin. Biol. Res 1989;314:659-671. [PubMed: 2608683]

Jones BW, Watt CB, Frederick JM, et al. Retinal remodeling triggered by photoreceptor degenerations. J. Comp. Neurol 2003;464:1-16. [PubMed: 12866125]

Kanuga N, Winton HL, Beauchène L, et al. Characterization of genetically modified human retinal pigment epithelial cells developed for in vitro and transplantation studies. Invest. Ophthalmol. Vis. Sci 2002;43:546-555. [PubMed: 11818403]

Klimanskaya I, Hipp J, Rezai KA, West M, Atala A, Lanza R. Derivation and comparative assessment of retinal pigment epithelium from human embryonic stem cells using transcriptomics. Cloning Stem Cells 2004;6:217-245. [PubMed: 15671670]

LaVail MM. Legacy of the RCS rat: impact of a seminal study on retinal cell biology and retinal degenerative diseases. Prog. Brain. Res 2001;131:617-627. [PubMed: 11420975]

Li LX, Turner JE. Inherited retinal dystrophy in the RCS rat: prevention of photoreceptor degeneration by pigment epithelial cell transplantation. Exp. Eye. Res 1988;47:911-917. [PubMed: 3215300]

Lin N, Fan W, Sheedlo HJ, Aschenbrenner JE, Turner JE. Photoreceptor repair in response to RPE transplants in RCS rats: outer segment regeneration. Curr. Eye. Res 1996;15:1069-1077. [PubMed: 8921247] 
Little CW, Castillo B, DiLoreto DA, et al. Transplantation of human fetal retinal pigment epithelium rescues photoreceptor cells from degeneration in the Royal College of Surgeons rat retina. Invest. Ophthalmol. Vis. Sci 1996;37:204-211. [PubMed: 8550325]

Lund RD, Adamson P, Sauve, et al. Subretinal transplantation of genetically modified human cell lines attenuates loss of visual function in dystrophic rats. Proc. Natl. Acad. Sci. USA 2001;98:9942-9947. [PubMed: 11504951]

Lund RD, Ono SJ, Keegan DJ, Lawrence JM. Retinal transplantation: progress and problems in clinical application. J. Leukoc. Biol 2003;74:151-160. [PubMed: 12885930]

Marc RE, Jones BW, Watt CB, Strettoi E. Neural remodeling in the retinal degeneration. Prog. Ret. Eye. Res 2003;22:607-655.

McGill TJ, Douglas RM, Lund RD, Prusky GT. Quantification of spatial vision in the Royal College of Surgeons rat. Invest. Ophthalmol. Vis. Sci 2004;45:932-6. [PubMed: 14985313]

McGill TJ, Lund RD, Douglas RM, Wang S, Lu B, Prusky GT. Preservation of vision following cellbased therapies in a model of retinal degenerative disease. Vis. Res 2004;44:2559-66. [PubMed: 15358071]

McGinnis JF, Stepanik PL, Chen W, Elias R, Cao W, Lerious V. Unique retina cell phenotypes revealed by immunological analysis of recoverin expression in rat retina cells. J. Neurosci. Res 1999;55:252260. [PubMed: 9972828]

Perlman I. Dark-adaptation in abnormal (RCS) rats studied electroretinographically. J. Physiol 1978;278:161-175. [PubMed: 671282]

Perlman I. Kinetics of bleaching and regeneration of rhodopsin in abnormal (RCS) and normal albino rats in vivo. J. Physiol 1978;278:141-159. [PubMed: 671279]

Peng YW, Senda T, Hao Y, Matsuno K, Wong F. Ectopic synaptogenesis during retinal degeneration in the royal college of surgeons rat. Neurosci 2003;119:813-820.

Pignatelli V, Cepko CL, Strettoi E. Inner retinal abnormalities in a mouse model of Leber's congenital amaurosis. J. Comp. Neurol 2004;469:351-359. [PubMed: 14730587]

Pinilla I, Lund RD, Sauvé Y. Contribution of rod and cone pathways to the dark-adapted electroretinogram (ERG) b-wave following retinal degeneration in RCS rats. Vis. Res 2004;44:2467-2474. [PubMed: 15358082]

Pinilla I, Lund RD, Lu B, Sauvé Y. Measuring the cone contribution to the ERG b-wave to assess function and predict anatomical rescue in RCS rats. Vis. Res 2005;45:635-641. [PubMed: 15621180]

Sauvé Y, Girman SV, Wang S, Keegan DJ, Lund RD. Preservation of visual responsiveness in the superior colliculus of RCS rats after retinal pigment epithelium cell transplantation. Neurosci 2002;114:389401.

Sauvé Y, Lu B, Lund RD. The relationship between full field electroretinogram and perimetry-like visual thresholds in RCS rats during photoreceptor degeneration and rescue by cell transplants. Vis. Res 2004;44:9-18. [PubMed: 14599567]

Sauvé Y, Pinilla I, Lund RD. Partial preservation of rod and cone ERG function following subretinal injection of ARPE-19 cells in RCS rats. Vis. Res 2006;46:1459-1472. [PubMed: 16364396]

Sheedlo HJ, Li L, Turner JE. Photoreceptor cell rescue at early and late RPE-cell transplantation periods during retinal disease in RCS dystrophic rats. J. Neural. Transplant. Plast 1991;2:55-63. [PubMed: 1868118]

Spencer B, Agarwala S, Gentry L, Brandt CR. HSV-1 vector-delivered FGF2 to the retina is neuroprotective but does not preserve functional responses. Mol. Ther 2001;3:746-756. [PubMed: 11356079]

Vaney DI. Retinal neurons: cell types and coupled networks. Prog. Brain Res 2002;136:239-254. [PubMed: 12143385]

Villegas-Pérez MP, Lawrence JM, Vidal-Sanz M, LaVail MM, Lund RD. Ganglion cell loss in RCS rat retina: a result of compression of axons by contracting intraretinal vessels linked to the pigment epithelium. J. Comp. Neurol 1998;392:58-77. [PubMed: 9482233]

Vollrath D, Feng W, Duncan JL, Yasumura D, D'Cruz PM, Chappelow A, Matthes MT, Kay MA, LaVail MM. Correction of the retinal dystrophy phenotype of the RCS rat by viral gene transfer of Mertk. Proc. Natl. Acad. Sci. USA 2001;98:12584-12589. [PubMed: 11592982] 
Wang S, Lu B, Lund RD. Morphological changes in the RCS retina during photoreceptor degeneration and after cell-based therapy. J. Comp. Neurol 2005;491:400-417. [PubMed: 16175546] 


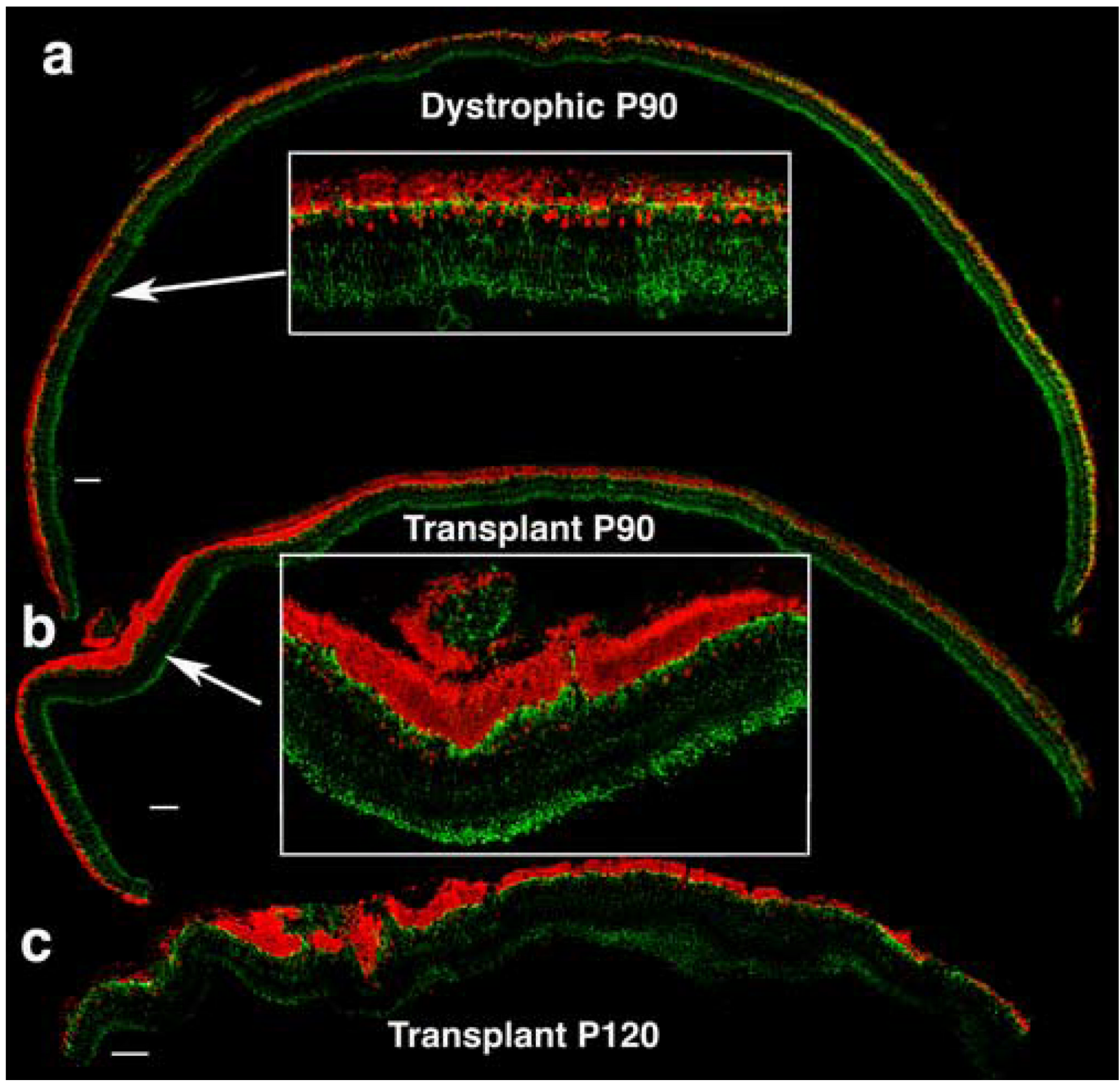

Figure 1.

Low magnification cross section of retinas double immunolabeled with antibodies against recoverin (red) and PKC (green). (a) Dystrophic RCS rat retina at P90 showing rod bipolar cells (green), two subtypes of cone bipolar cells (red) and one row of photoreceptors. (b) P90 transplanted retina showing well preserved photoreceptors in transplant area (inset) diminishing with the eccentricity. (c) P120 RCS rat transplanted retina. The preservation area extends $2 \mathrm{~mm}$ from the injection area. Scale bar represents $100 \mu \mathrm{m}$. 

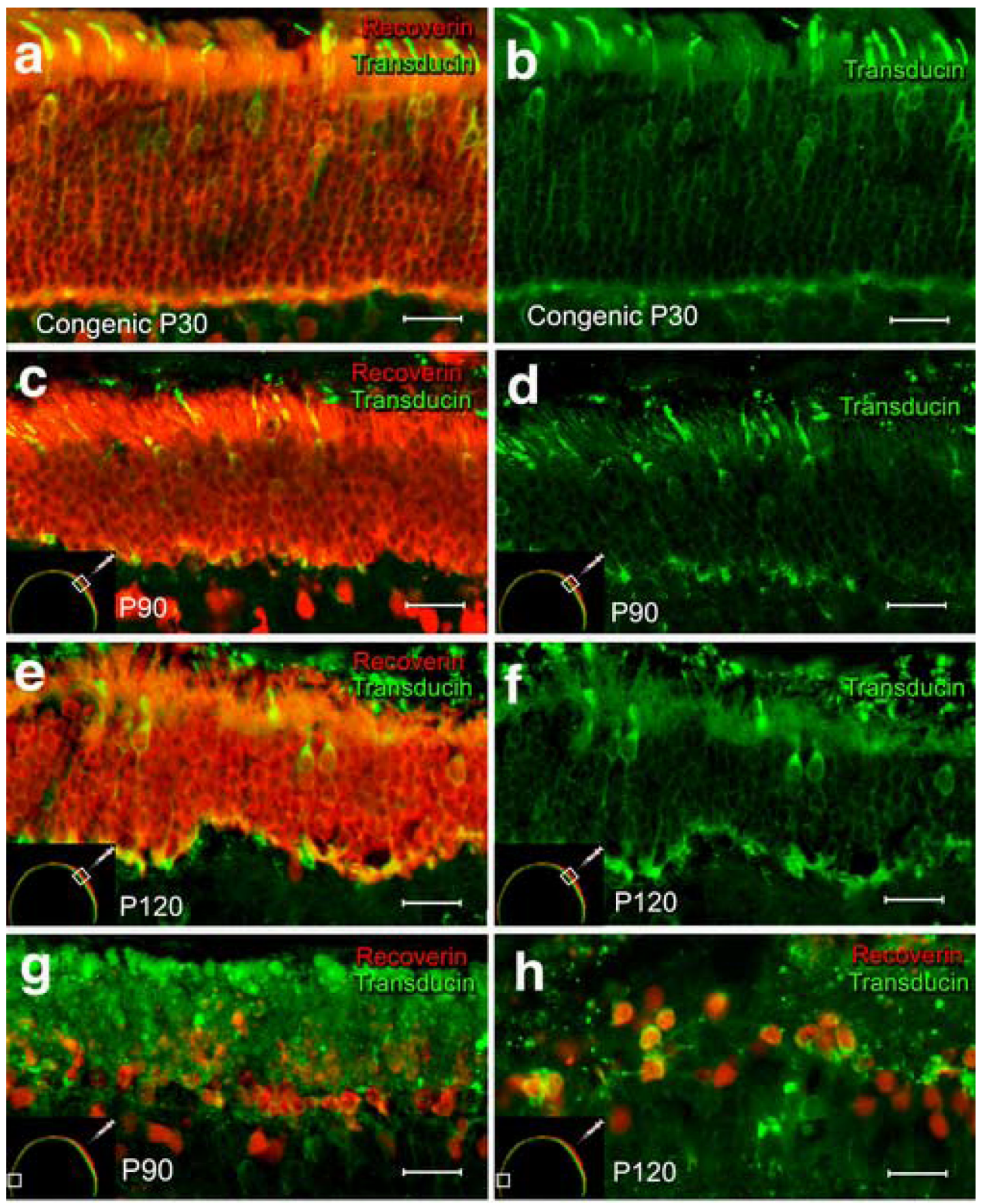

Figure 2.

Effect of hRPE cell transplantation on photoreceptor morphology. Cross sections of retinas from congenic rat at P30 (a, b), and dystrophic transplanted retina (P90, P120). Recoverin immunostained cones and rods (red) and transducin immunostained cones (green). Note that recoverin also stains cone bipolar cells type 2 and 8 (McGinnis et al., 1999), while transducin is specific to cones only. $(a, b)$ Rows of photoreceptors showing well developed cones and rods in congenic rat. Transducin staining (green channel) shows cone morphology. (c, d) Cones and rods in transplant area at P90 showing well preserved photoreceptors. In the area far from the transplant (g) only one row of photoreceptors was preserved. (e, f) Transplant area from an RCS rat at P120. Rows of photoreceptors can be observed compared with only one row of 
cones (yellow) in area distant to transplant area where there is also a debris zone (h). Inset shows the injection area indicated by a syringe; the square shows the area where the picture was taken. Scale bar represents $20 \mu \mathrm{m}$. 

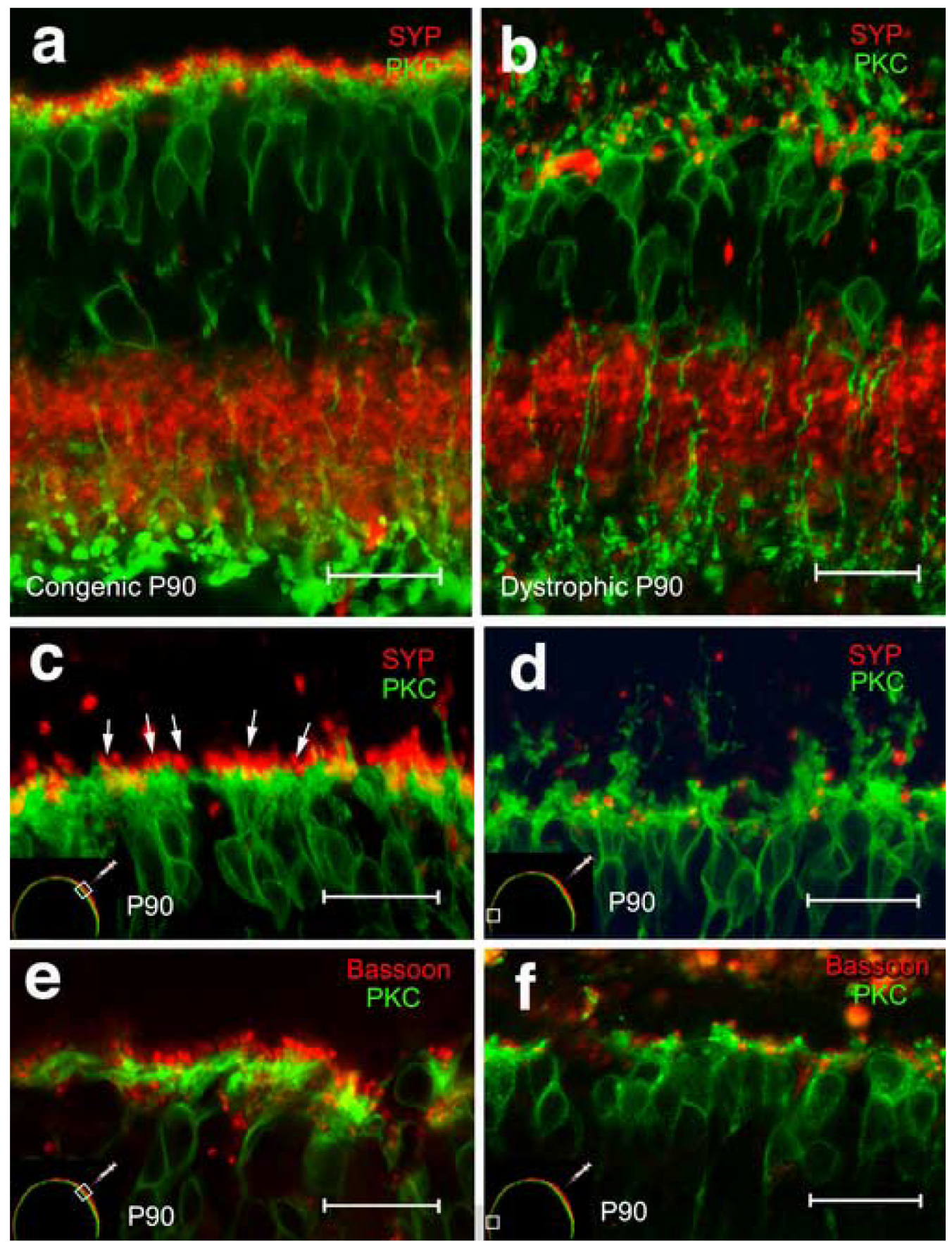

Figure 3.

Preservation of synaptic contacts of bipolar cells and bipolar cell sprouting. (a) Double immunolabeled with synaptophysin (red) and PKC (green) in congenic P30 RCS rats showing synaptic contacts between photoreceptor terminals and well developed rod bipolar dendrites. (b) Dystrophic RCS rats at P90 show no synaptic contacts and sprouting of bipolar dendrites into the debris zone. (c) In transplanted rats at P90, synaptic contacts between bipolar cell dendrites and photoreceptor terminals are observed in the OPL. (d) Lack of synaptic contacts in OPL in areas distant from the injection. There was no bipolar cell sprouting in the transplant area (c) compared to dystrophic rat at P90 (b). Short dendrites emerging from the cell bodies indicting an initial of sprouting were found in the opposite side of the retina from the transplant 
(d). (e) Double immunolabeling with antibodies against PKC (green) and bassoon (red) showing preservation of synaptic contacts between rod bipolar cells and photoreceptor terminals in injection area compared with areas away from the injection site (f) where very few synaptic contacts can be recognize. Scale bar represents $20 \mu \mathrm{m}$. 


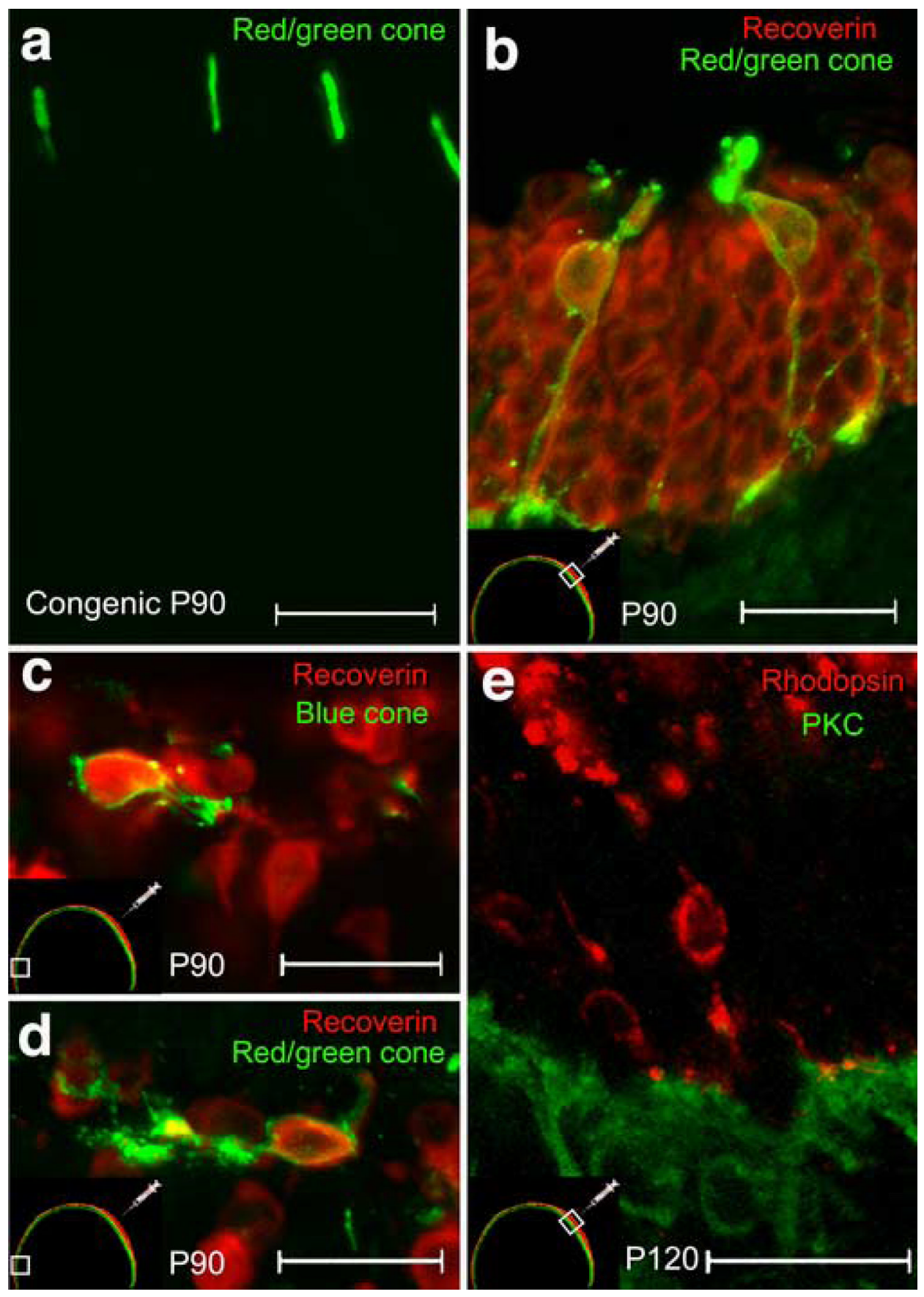

Figure 4.

Cross sections of retinas using antibody against green-red opsins (green) (a) showing normal cone outer segments in congenic P30 retina. (b) Double immunostaining with antibodies against recoverin (red) and green-red opsins (green). Cone morphology is partially preserved in the injection area compared with fragmented outer segments inside debris zone in areas far from the transplant (c). (d) Double immunostaining with antibodies against recoverin (red) and anti blue opsin antibody (green) showing the abnormal morphology of a blue cone in area far from the injection site. (e) Rhodopsin antibody stains rod outer segments and a few cell bodies and axon terminals in the transplant area. PKC- $\alpha$ (green) staining rod bipolar cells. Scale bar represents $20 \mu \mathrm{m}$. 

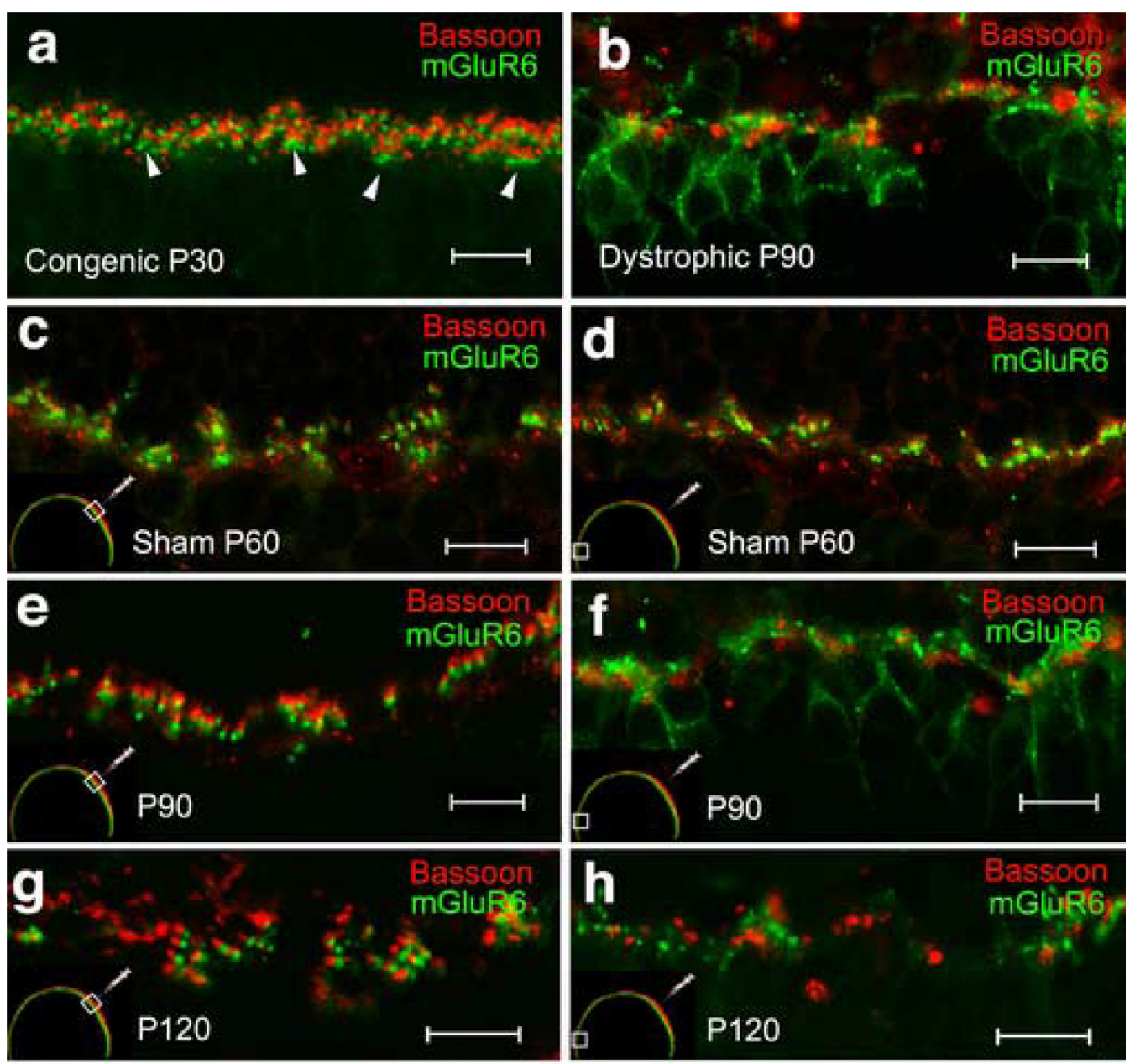

Figure 5.

Confocal fluorescence micrographs of retinal cross sections showing the preservation of photoreceptor ribbon synapses with bipolar cell dendrites in the OPL of transplanted retinas. The synaptic ribbons were visualized with an antibody against the protein, bassoon, (red) which labels photoreceptor ribbons in both cone pedicles and rod spherules. Bipolar dendritic tips are labeled with an antibody against mGluR6. (a) One to two rows of synaptic pairs of bassoon and mGluR6 profiles were identified in P30 congenic rat: arrows indicate synaptic contacts between cones pedicles and cone bipolar cells. (b) In P90 RCS rat, the paired bassoon/mGluR6 profiles in the OPL disappear. Bipolar cell bodies express mGluR6 and the number of bassoonmGluR6-labeled ribbons has already decreased. (c, d) Sham injected RCS rats at P60 showed some pairs in a discontinuous layer in areas close to the injection and far to the injection site. (e) In the transplant area at P90, one row of paired bassoon/mGluR6 profiles remains indicating preservation of synaptic order compared with areas distant to injection in the same retina (f). Even at P120, some pairs of synaptic contacts between photoreceptors and bipolar cells were observed in injection area $(\mathrm{g})$. (h) No evident pairs were observed away from the injection site at P120. Scale bar: (a-f) $10 \mu \mathrm{m} ;(\mathrm{g}-\mathrm{h}), 20 \mu \mathrm{m}$. 

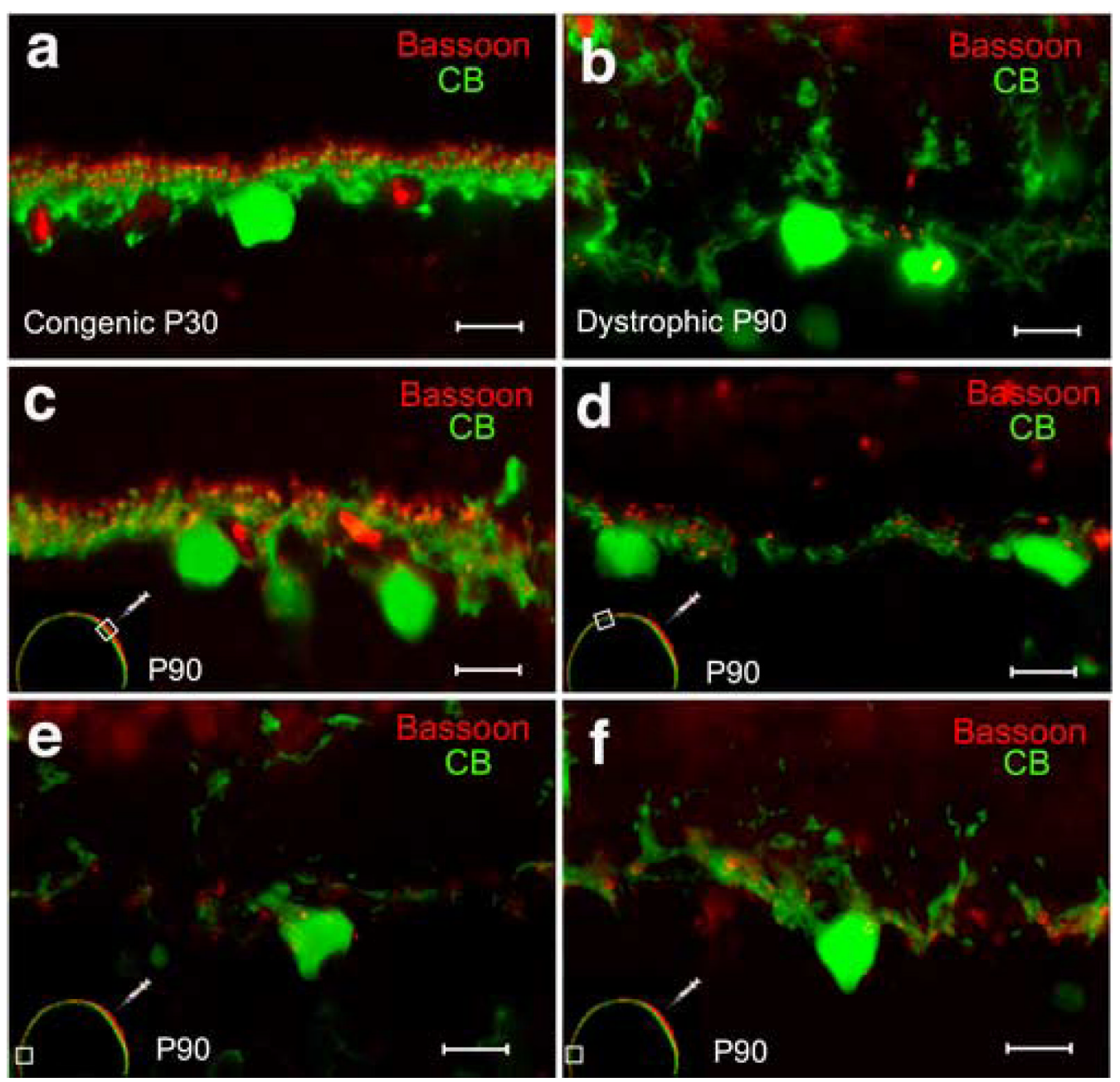

Figure 6.

Effects of hRPE cell injection on synaptic changes between horizontal cells and photoreceptors. (a) Confocal micrograph of horizontal cells visualized by calbindin immunocytochemistry (green) and photoreceptors synaptic ribbons identified by bassoon immunoreactivity (red), showing their paired synaptic contacts in P30 congenic rats. In P90 dystrophic retinas (b) horizontal cell processes show no contacts with bassoon-labeled profiles and extensive sprouting into the ONL. In P90 transplanted retina area (c) the synaptic contacts are well preserved with a little preservation in areas distant to the injection (d) and far away (e, f) where horizontal cell terminals and bassoon immunoreactivity have almost disappeared. Scale bar represents $10 \mu \mathrm{m}$. 

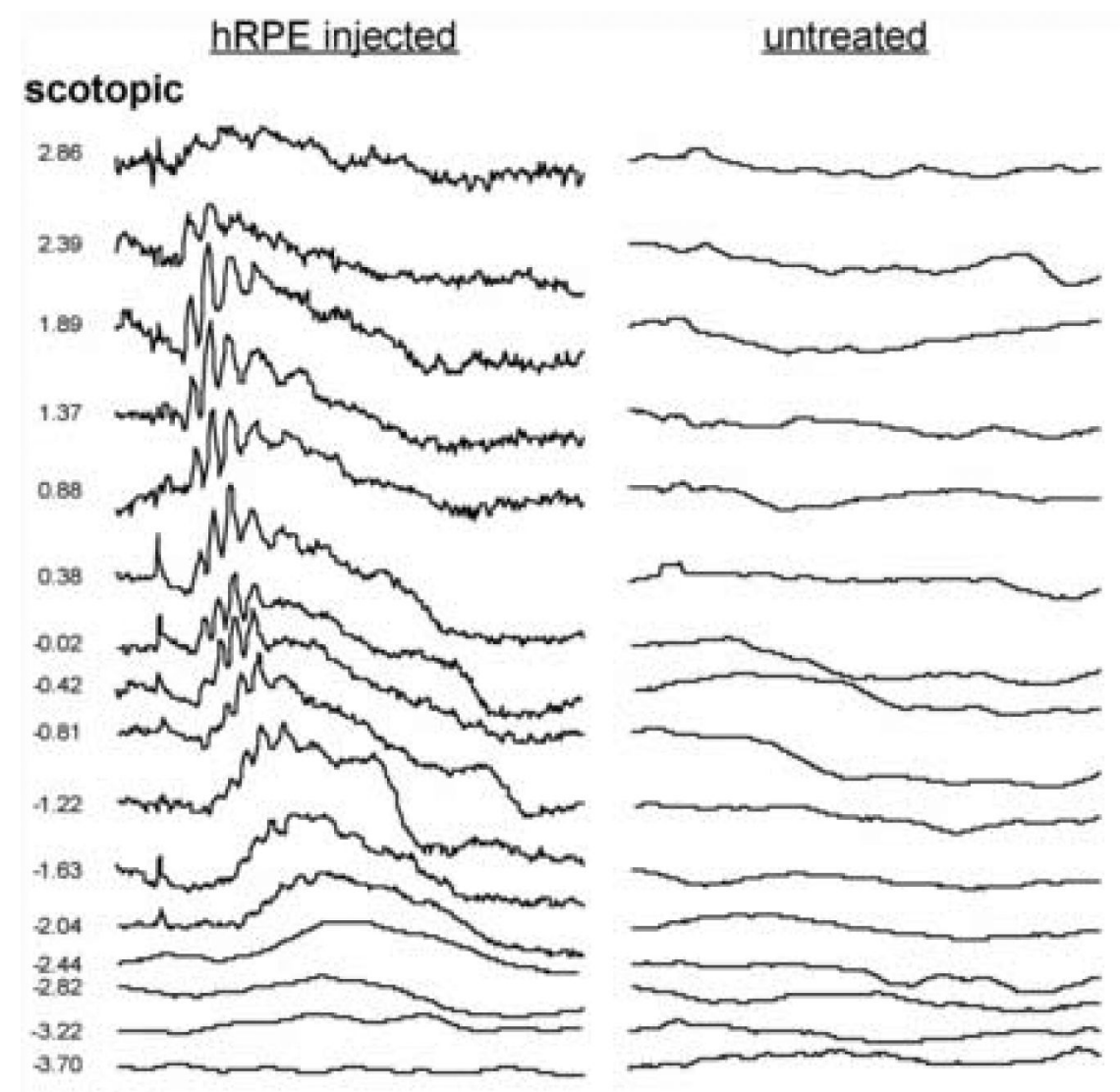

\section{photopic}
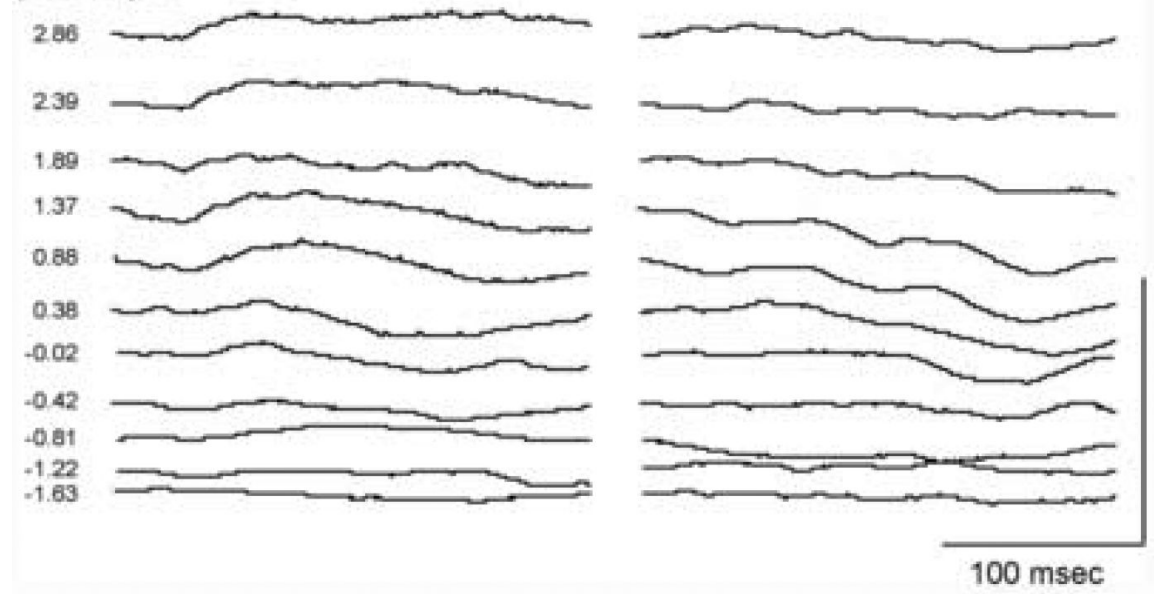

Figure 7.

Example of ERG traces from an animal treated with preventive subretinal injections of hRPE cells and from age-matched untreated animal, both at age P90. Flash presentation is at $10 \mathrm{msec}$ from the beginning of each trace. Stimulus intensity values (numbers on left of traces) are in $\log \mathrm{cds} / \mathrm{m}^{2}$. 
Table 1

\begin{tabular}{|c|c|c|c|}
\hline Antigen & Antiserum & Source & Dilution \\
\hline Bassoon & Mouse anti-bassoon & Stressgen & 1: 5000 \\
\hline Blue-opsin & Rabbit anti-opsin blue & Chemicon & $1: 500$ \\
\hline Calbindin & Rabbit anti-CB & Swant & $1: 500$ \\
\hline Green-red opsin & Rabbit anti-opsin G-R & Chemicon & $1: 500$ \\
\hline Metabotropic glutamate receptor 6 & Rabbit anti-mGluR6 & Neuromics & $1: 3000$ \\
\hline Protein Kinase $\mathrm{C} \alpha$ & Rabbit anti-PKC & Santa Cruz & $1: 100$ \\
\hline Protein Kinase $\mathrm{C} \alpha$ & Mouse anti-PKC & Santa Cruz & $1: 100$ \\
\hline Recoverin & Mouse anti-recoverin & Dr. McGinnis & $1: 5000$ \\
\hline Recoverin & Rabbit anti-recoverin & Dr. McGinnis & $1: 20000$ \\
\hline Rhodopsin & Mouse anti-rhodopsin & Dr. Molday & $1: 100$ \\
\hline Synaptophysin & Mouse anti- synaptophysin & Sigma & $1: 300$ \\
\hline$\gamma$-Transducin & Rabbit anti- $\gamma$-transducin & Cytosignal & $1: 500$ \\
\hline
\end{tabular}

